\title{
Probing the Spontaneous Emission Dynamics in Si-Nanocrystals-Based Microdisk Resonators
}

\author{
A. Pitanti, ${ }^{1, *}$ M. Ghulinyan, ${ }^{2}$ D. Navarro-Urrios, ${ }^{3}$ G. Pucker,${ }^{2}$ and L. Pavesi ${ }^{1}$ \\ ${ }^{1}$ Nanoscience Lab., Department of Physics, University of Trento, via Sommarive 14, Povo (TN), Italy \\ ${ }^{2}$ Advanced Photonics \& Photovoltaics, Fondazione Bruno Kessler, via Sommarive 18, Povo (TN), Italy \\ ${ }^{3}$ MIND-IN2UB, Departament d'Electrònica, Universitat de Barcelona, Carrer Martì i Franquès 1, 08028 Barcelona, Spain
}

(Received 7 July 2009; revised manuscript received 9 February 2010; published 9 March 2010)

As a possible cavity quantum electrodynamical system, unlike III-V quantum dots, Si-NCs are not considered ideal emitters for emission rate enhancement observations (Purcell effect). Here, we report on direct measurements of spontaneous emission rate enhancement of Si-NCs embedded in a whisperinggallery mode resonator at room temperature. Using time-resolved microphotoluminescence experiments, we demonstrate important lifetime reductions $(\sim 70 \%)$ for Si-NCs coupled to cavity modes with respect to uncoupled ones. Comparing experiments with the theoretical Purcell enhancement in a bad emitter regime, we estimate effective linewidths of $\sim 10 \mathrm{meV}$ through which Si-NC emitters are coupled to cavity photons. Finally, our study provides an alternative method for the estimation of subnatural linewidths of quantum dots at room temperature.

DOI: 10.1103/PhysRevLett.104.103901

Since the first demonstration of optical gain at optical wavelengths [1], nanocrystalline silicon has triggered a lot of scientific interest for its applications as a CMOScompatible optoelectronic material [2]. Integrated photonic components containing Si-NCs, in particular, resonator devices, have been realized and studied so far [3-6]. Key optical properties of single nanocrystals remain unaddressed, on one side, because of the difficulty to perform single-nanocrystal spectroscopic experiments; different fabrication techniques produce multi-dispersed-size nanocrystal ensembles. On the other side, the quantum-confined electronic structure of Si-NCs is far more complex than those of III-V compound quantum dots, due to the indirect band gap nature of the bulk silicon itself. Moreover, as initially proposed for nanostructured porous silicon, the quantum exchange interaction splits the excitonic state into few meV separated ( $\Delta \sim 10-20 \mathrm{meV}$, [7]) singlet (upper level) and triplet states (lower level) [8]. Thermal averaging of these two possible recombination paths results in a temperature-dependent radiative recombination probability, $\tau_{r}^{-1}=\left[3 / \tau_{L}+\left(1 / \tau_{U}\right) e^{-\Delta / k T}\right] /\left(3+e^{-\Delta / k T}\right)$ [9], where $\tau_{U}\left(\tau_{L}\right)$ is the upper-singlet (lower-triplet) radiative lifetime, $k$ is the Boltzmann constant, and $T$ is the temperature. Low-temperature photoluminescence (PL) experiments allow one to probe only the triplet recombination rate, being $\tau_{r} \sim \tau_{L}$. While at room temperature (RT) singlet transitions are dominant [10], no information can be extracted for these due to important nonradiative recombinations.

Interestingly, field-matter interactions can deeply modify the spontaneous emission rate $\left(\Gamma_{r}\right)$ of an emitter, in general, and of Si-NCs, in particular. This can occur, for example, coupling resonantly $\mathrm{Si}-\mathrm{NC}$ excitons to optical cavity modes; the presence of large photonic density of states can shorten the radiative lifetime, $\tau_{r}\left(\sim \Gamma_{r}^{-1}\right)$, of emitters (Purcell effect) [11]. Even if Si-NCs are not ideal
PACS numbers: 42.60.Da, 42.50.Pq, 78.67.Bf

candidates for studying the Purcell effect, the possibility to measure emission rate enhancement $\epsilon$ would provide useful information on the effective average linewidths through which $\mathrm{Si}-\mathrm{NC}$ emitters are coupled to resonator modes. The importance of such results sounds critical in view of the lack of experimental data for the electronic linewidth of $\mathrm{Si}-\mathrm{NCs}$ in bulk samples with broad size dispersion of emitters [12].

In this Letter we report on the direct estimation of Purcell enhancement for Si-NCs coupled to WGM of a microdisk resonator through RT time-resolved measurements. The emission lifetimes $(\tau)$ of Si-NCs are measured to be up to $70 \%$ shorter at wavelengths resonant with the WGMs (coupled) with respect to $\tau$ 's probed at out-ofresonance wavelengths (uncoupled). Such a difference in $\tau$ leads to relatively low Purcell factors as expected in the bad emitter regime, when the emitter homogeneous linewidth $\Delta \omega_{\mathrm{em}}$ is larger than that of the cavity $\left(\Delta \omega_{\text {cav }}\right)$. We show that such estimated linewidths imply upper limits for cavity quality factors $Q$ that could be utilized for efficient Purcell enhancement in Si-NCs. Moreover, we predict that large Purcell factors can be still probed using resonators with ultrasmall cavity mode volumes. Finally, the used method can be applied to other systems where fundamental linewidths are unknown or which are in the bad-coupling regime.

The Purcell enhancement factor $F_{P}$ represents the cavity figure of merit and for an ideal emitter reads as $F_{P}=$ $\frac{3}{4 \pi^{2}} \frac{Q}{V_{m}} \tilde{\lambda}^{3}[Q$ is the cavity quality factor, $\tilde{\lambda}$ is the wavelength in the dielectric material $(\tilde{\lambda}=\lambda / n)$, and $V_{m}$ is the mode volume] [15]. The ideality condition implies that (i) the emitter and the cavity resonances are spectrally tuned and $\Delta \omega_{\text {em }} \ll \Delta \omega_{\text {cav }}$, (ii) the emitted field is aligned with that of the cavity and placed in a cavity-field antinode, and (iii) the nonradiative recombination rate is negligible. Often, the Purcell enhancement is estimated as the ratio 
of measured PL intensities from a cavity resonance and the bulk material. Even if the PL intensity is directly proportional to the radiative recombination rate, this approach, however, results in strongly overestimated $F_{P}$ 's; geometrical correction effects have to be taken into account when the WGM cavity emission (usually within a small solid angle) and the bulk emission (isotropic) are compared [16]. The degree of uncertainty by which the correction factors can be estimated, allows one to extract the Purcell enhancement only by comparing lifetimes for an emitter coupled and for an emitter not-coupled (uncoupled) with a cavity mode, as previously done for III-V quantum dots in $[15,17]$.

We have realized different Si-NC-doped WGM resonators (for fabrication details see [3]). We used a hydrogen passivation treatment to increase the emission intensity [18]. We have fabricated both flat ( $\mu$-disk, Fig. 1(a), right) and bent ( $\mu$-kylix, Fig. 1(a), left) resonators. While the dimensions and the amount of the active material in both type of disks are the same, the bent disks provide highest $Q$ 's in a spectral range which is blueshifted by $\sim 60 \mathrm{~nm}$ with respect to the flat ones [19]. Figures 1(b) and 1(c) summarize the necessary optical characteristics for the estimation of $F_{P}$; subnanometer WGM resonances [Fig. 1(b)] for the quasi-TM polarization have been probed by means of cw micro-PL experiments (for details see [3]). $F_{P}$ has been estimated using the experimental Q's and mode volumes [Fig. 1(d)], calculated through an FDTD package [20] [Fig. 1(c)].

The PL lifetimes have been measured through timeresolved experiments using the $488 \mathrm{~nm}$ line of a $\mathrm{kHz}-$ modulated cw laser source. At first, we measured lifetimes in spectral dips between neighboring WGM resonances, where the excitons are considered to be uncoupled to any cavity mode. The decays have been fitted by a stretchedexponential function, $I(t) / I_{0}=e^{-[(t-t 0) / \tau]^{\beta}}$, which ac-

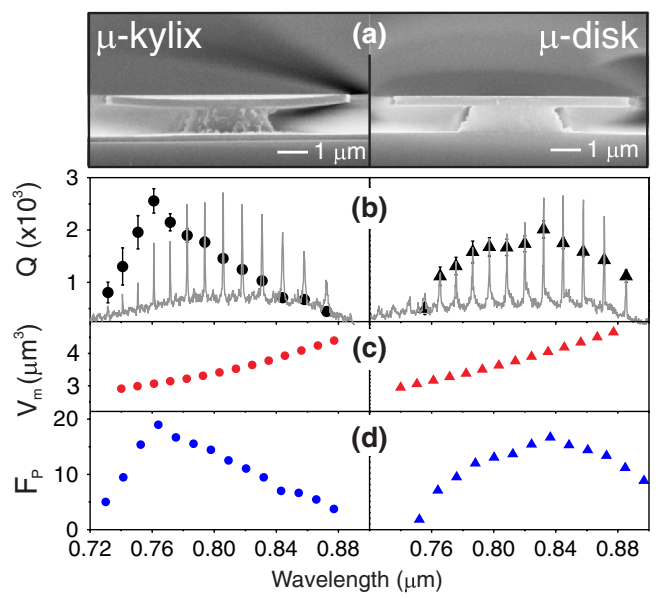

FIG. 1 (color online). (a) Cross-sectional scanning electron microscopy images of microdisk resonators. (b) PL intensity of quasi-TM WGMs with the calculated quality factors $(\boldsymbol{O}, \boldsymbol{\nabla})$. (c) Numerically calculated mode volumes. (d) The calculated cavity figure-of-merit-ideal Purcell factor $F_{P}$. counts for interparticle hopping phenomena of excitons in Si-NCs [21] and emission-detection frequency detuning effects. The stretched-exponential function represents a distribution of curves with different lifetimes [Kohlrausch-Williams-Watts (KWW) distribution]. The parameters $\tau$ and $\beta$ allow one to extract the average PL lifetime as the first order moment, $\tau_{\mathrm{PL}}=\tau / \beta G[1 / \beta]$, where $G[]$ is the Gamma function. We observe that $\tau_{\mathrm{PL}}$ shortens at higher emission energies [see Fig. 4(a)], as expected from high crystalline quality samples [22].

Next, we measured PL lifetimes at cavity peak wavelengths. Since our emitters are uniformly distributed in the resonator, the PL emission from peaks results from a contribution of (i) photons coupled to the cavity mode and (ii) a background signal of emitters uncoupled to the WGMs (leaky contribution) [Fig. 2(a)] [16]. In this scheme, we fit the decays employing the expression

$$
I(t) / I_{0}=A e^{-\left[(t-t 0) / \tau_{\text {peak }}\right]^{\beta}}+e^{-\left[(t-t 0) / \tau_{\text {dip }}\right]},
$$

where the term $A$ stands for the intensity ratio of coupled and uncoupled emitters and has been determined from static PL measurements shown in Figs. 2(b) and 2(c). In Eq. (1) $\tau_{\text {peak }}$ is the lifetime of mode-coupled emitters, while $\tau_{\text {dip }}$ represents the average lifetime at the peak wavelength extracted from interpolated $\tau_{\mathrm{PL}}$ data for dips (and, thus, represents the uncoupled emitters lifetime). Figure 3 reports an example of measured peak and dip lifetimes, where important differences can be appreciated. The extracted lifetimes at various resonances and dips in a wide spectral range are summarized in Fig. 4(a). Before addressing the Purcell enhancement, we first consider the implication of the stretched-exponential decay in conditions of bad emitter regime. For simplicity, neglecting the nonradiative recombination rates, the total emission rate

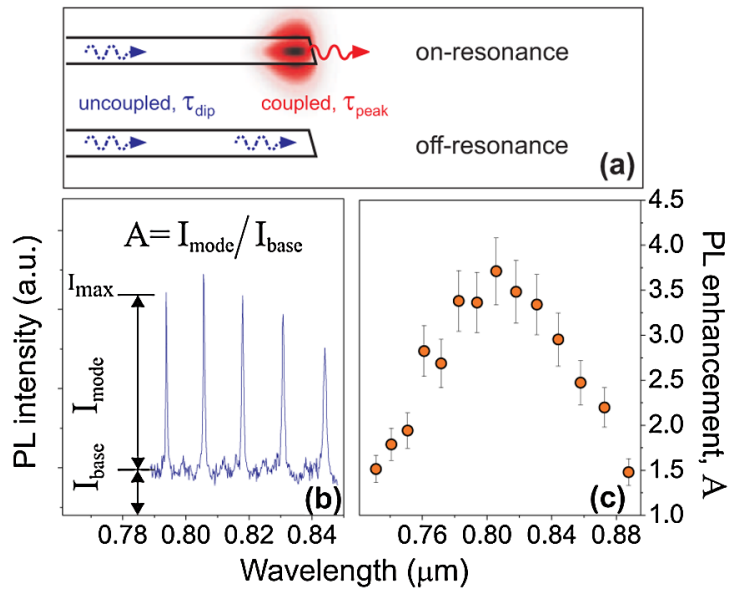

FIG. 2 (color online). (a) Graphical sketch explaining the use of exponential decay functions for on- and off-resonance conditions. (b) The fitting parameter $A$ in Eq. (1) has been defined as the PL intensity ratio of the mode $I_{\text {mode }}$ and the background $I_{\text {base }}$. (c) The extracted values of parameter $A$ from the PL spectra of Fig. 1(b). 


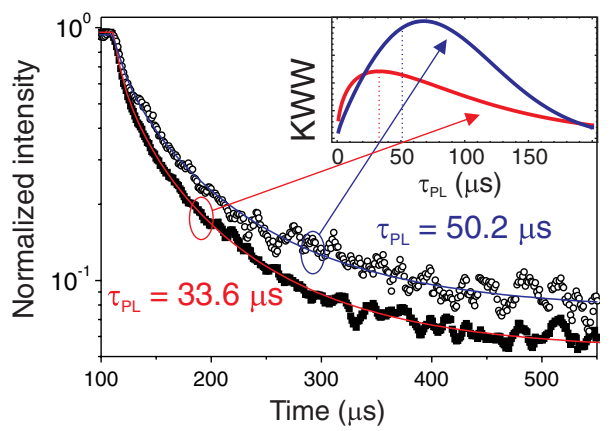

FIG. 3 (color online). Measured out-of-resonance (full squares, $\lambda=853 \mathrm{~nm}$ ) and on-resonance (empty circles, $\lambda=$ $846 \mathrm{~nm})$ PL signals. The data have been fitted using single and double stretched exponentials, respectively. The inset shows the associated KWW lifetime distributions.

from a single nanocrystal at the frequency $\omega$ becomes

$$
\Gamma(\omega)=\Gamma_{r}\left[1+A f(\omega) \tilde{F}_{P} \delta\left(\omega-\omega_{\text {cav }}\right)\right],
$$

where the function $f=\frac{\Delta \omega_{\mathrm{em}}^{2}}{\Delta \omega_{\mathrm{em}}^{2}+4\left(\omega-\omega_{\mathrm{cav}}\right)^{2}}$ describes the spectral detuning of the emitter from the cavity. The latter has been considered as a Dirac $\delta$ function. With this result the PL decays of an ensemble of $N$ emitters, each one characterized by its own frequency $w_{i}$ and radiative rate $\Gamma_{i}$, can be described as the sum

$$
\sum_{i}^{N} \frac{\Delta \omega_{\mathrm{em}}^{2}}{\Delta \omega_{\mathrm{em}}^{2}+4\left(\omega-\omega_{i}\right)^{2}}\left[1+A \delta\left(\omega_{i}-\omega_{\mathrm{cav}}\right)\right] e^{-\Gamma_{i} t} .
$$

Equation (3) tends to a stretched-exponential decay with different sets of $(\beta, \tau)$ depending on the detection frequency (spectral dip or peak). The spectral detuning effects, in fact, are included in the KWW distribution

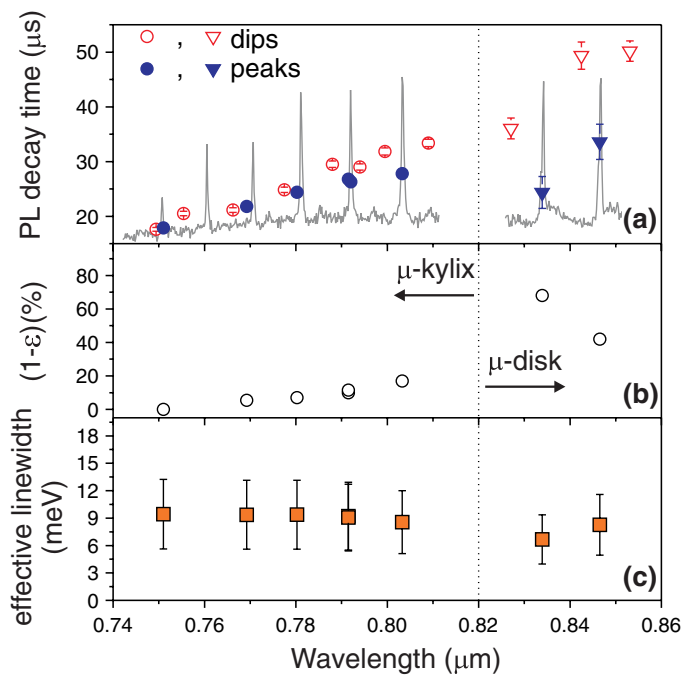

FIG. 4 (color online). (a) Measured PL lifetimes for the peaks $(\boldsymbol{\nabla}, \boldsymbol{\nabla})$ and dips $(\bigcirc, \nabla)$ of the WGM spectrum. (b) Calculated lifetime shortening $\epsilon=\frac{\tau_{\text {dip }}}{\tau_{\text {peak }}}$ and (c) the estimated Si-NC effective linewidth at room temperature. parameters and thus are already considered during the fitting procedure of PL decays [23]. We note that the stretched-exponential decay is a peculiarity of the bad emitter regime, whereas in the limit of $\Delta \omega_{\mathrm{em}} \ll \Delta \omega_{\mathrm{cav}}$, such as in the case of III-V quantum dots, usually single exponentials are observed [17]. Regarding our experiment, at room temperature, the nonradiative recombination rate cannot be neglected. Therefore, we have calculated the ratio $\epsilon=\tau_{\text {dip }} / \tau_{\text {peak }}$, which is a function of radiative, $\tau_{r}$, and nonradiative lifetimes, $\tau_{n-r}^{-1}=\tau_{\text {dip }}^{-1}-\tau_{r}^{-1}$.

With this, $\epsilon=1+\frac{\tau_{\text {dip }}}{\tau_{r}}\left(\tilde{F}_{P}-1\right)$. Here $\tilde{F}_{P}$ is the nonideal Purcell factor, which has been estimated from empirical considerations following [17]:

$$
\tilde{F}_{P}=g \frac{3}{4 \pi^{2}} \frac{\tilde{\lambda}^{3}}{V_{m}}\left(\frac{1}{Q_{\mathrm{cav}}}+\frac{\Delta \omega_{\mathrm{em}}}{\omega_{\mathrm{em}}}\right)^{-1} f(\langle\omega\rangle),
$$

where the clockwise-anticlockwise mode degeneracy $g$ has been considered $(g=2)$. The term $\left(1 / Q_{\text {cav }}+\Delta \omega_{\text {em }} / \omega_{\text {em }}\right)$ represents the inverse of an effective quality factor, taking into account both the cavity mode and emitter linewidths. Spatial and polarization averaging effects have been evaluated employing Eq. (1) [24], where the contribution from leaky modes has been eliminated [15]. While the spectral detuning effects are already included in the $(\beta, \tau)$ set of the stretched-exponential distribution, in Eq. (4) we have considered an additional Lorentzian-shaped term [15], $f(\langle\omega\rangle)$, which accounts for the finite spectral resolution of the detecting system [25].

While the difference between peak and dip lifetimes is appreciable, the resulting ratio $\epsilon$ is slightly larger than unity [Fig. 4(b)], with a maximum relative shortening (1 $\epsilon$ ) of about $70 \%$. Note that $\epsilon$ represents the lower limit of $\tilde{F}_{P}$ when nonradiative recombination rates are nonnegligible, as in our case. On the other hand, when $\Gamma_{n-r} \rightarrow$ 0 , the values of $\epsilon$ and $\tilde{F}_{P}$ coincide $\left(\lim _{\tau_{n-r} \rightarrow \infty} \epsilon=\tilde{F}_{P}\right)$. In the expression for $\epsilon$, the radiative lifetime in our hydrogenpassivated material has been attributed to the singlet state [26]. In fact, the $\mathrm{H}_{2}$ passivation is known to quench the triplet state recombination rate in Si-NCs [18]. Comparing $\epsilon$ and $\tilde{F}_{P}$, we extract effective linewidths $(\sim 10 \mathrm{meV})$ for spontaneous emission enhancement [Fig. 4(c)].

The effective linewidth describes the average nanocrystal-resonant-cavity-field coupling, and is expected to be of the same order of Si-NC's homogeneous electronic linewidth. The literature does not report any data for this last in PECVD-grown Si-NCs; therefore, we compare our results with those reported for other material systems containing Si-NC. Because of their reduced size, $\mathrm{Si}-\mathrm{NC}$ can show breakdown of the $\vec{k}$-conservation rule in optical transitions [27]. For high quantum confinement energies, the no-phonon (NP) quasidirect transition probability starts to rule out the phonon-assisted (PA) indirect one, which represents the main radiative recombination path in bulk Si. While PA transitions show temperaturedependent linewidth broadening, NP ones are considered energetically subnatural (narrower than $k T$ ) [13]. This has 
been confirmed in low- $T$ spectroscopic experiments on tens of nm-sized single $\mathrm{Si}$ nanopillars [13], where a $2 \mathrm{meV}$ narrow linewidith at $35 \mathrm{~K}$ has been measured. As predicted in Ref. [27], the NP to PA transitions ratio should be stronger in $\mathrm{SiO}_{2}$-embedded $\mathrm{Si}$-NCs of a few $\mathrm{nm}$ in size with respect to nanoparticles obtained from mesoporous $\mathrm{Si}$ oxidation $(\sim 10 \mathrm{~nm})$. In addition, quenching of the triplet state recombination through $\mathrm{H}_{2}$ passivation should cause a further narrowing of the linewidth.

A measured linewidth of $10 \mathrm{meV}$ corresponds to emitter $Q$ factors, $Q_{\mathrm{em}}=\omega_{\mathrm{em}} / \Delta \omega_{\mathrm{em}}$, of the order of 200 . These Q's impose an upper limit on the cavity linewidth which can be efficiently exploited to couple Si-NCs to resonator modes. A larger $Q$ factor does not affect the radiative lifetime but simply filters out the emission. However, the Purcell enhancement can be still favored by a small mode volume of the cavity [28] (in our resonators, for example, $\left.V_{m} \sim 25 \tilde{\lambda}^{3}\right)$. Utilizing ultrasmall mode volumes $\left(V_{m} \sim\right.$ $\tilde{\lambda}^{3}$ ), such as those of photonic crystal nanocavities [29], Purcell enhancements of $\tilde{F}_{P} \sim 25$ can be expected.

To conclude, we report RT spectroscopic measurements of the spontaneous emission rate modification of Si-NCs in an optical cavity. Direct time-resolved PL experiments demonstrate that a small Purcell enhancement can be observed even in the bad emitter regime, when the emitter homogeneous linewidth is larger than that of the cavity. We show that while the possibility to observe large $\tilde{F}_{P}$ employing high- $Q$ cavities is limited by the estimated effective linewidths for Si-NC coupling, strong Purcell enhancement can still be reached embedding nanocrystals in ultrasmall mode volume cavities. Finally, this method can be further generalized for a wide class of cavity-embedded emitters for which important optical properties, such as linewidth, are not known.

The authors wish to thank I. Carusotto, D. Sarchi, L. C. Andreani, and D. Gerace for stimulating discussions and A. Marconi for technical help. We also would like to thank the referees for useful comments which greatly improved the manuscript.

*pitanti@science.unitn.it

[1] L. Pavesi, L. Dal Negro, C. Mazzoleni, G. Franzò, and F. Priolo, Nature (London) 408, 440 (2000).

[2] Zh. Yuan et al., Proc. IEEE 97, 1250 (2009).

[3] M. Ghulinyan et al., Opt. Express 16, 13218 (2008).

[4] A. M. Beltaos and A. Meldrum, J. Lumin. 126, 607 (2007).

[5] R.-J. Zhang et al., Appl. Phys. Lett. 88, 153120 (2006).

[6] T.J. Kippenberg et al., Phys. Rev. Lett. 103, 027406 (2009).

[7] A. Sa'ar, J. Nanophoton. 3, 032501 (2009).
[8] P. D. Calcott, K. J. Nash, L. T. Canham, M. J. Kane, and D. Brumhead, J. Phys. Condens. Matter 5, L91 (1993).

[9] O. Bisi, S. Ossicini, and L. Pavesi, Surf. Sci. Rep. 38, 1 (2000).

[10] At high temperature, the PL dynamics is governed by thermal-weighted mixing of two transitions, resulting in much favorable singlet (parity-allowed) transitions with respect to triplet (parity-forbidden) ones.

[11] E. M. Purcell, Phys. Rev. 69, 681 (1946).

[12] Few existing studies deal with $e$-beam lithographed topoxidized Si nanopillars (Ref. [13]) and porous silicon grains deposited from diluted colloidal suspensions (Ref. [14]): in both cases the nanocrystalline system is different from what we study here-Si-NCs embedded in a $\mathrm{SiO}_{2}$ matrix, grown through plasma enhanced chemical vapour deposition (PECVD).

[13] I. Sychugov, R. Juhasz, J. Valenta, and J. Linnros, Phys. Rev. Lett. 94, 087405 (2005).

[14] J. Martin, F. Cichos, F. Huisken, and C. von Borczyskowski, Nano Lett. 8, 656 (2008).

[15] J. M. Gérard et al., Phys. Rev. Lett. 81, 1110 (1998).

[16] B. Gayral and J. M. Gérard, Phys. Rev. B 78, 235306 (2008).

[17] J. M. Gérard and B. Gayral, J. Lightwave Technol. 17, 2089 (1999).

[18] S. Godefroo et al., Nature Nanotech. 3, 174 (2008).

[19] M. Ghulinyan, A. Pitanti, G. Pucker, and L. Pavesi, Opt. Express 17, 9434 (2009).

[20] A. Farjadpour et al., Opt. Lett. 31, 2972 (2006).

[21] L. Pavesi and M. Ceschini, Phys. Rev. B 48, 17625 (1993).

[22] M. Fujii, K. Imakita, K. Watanabe, and S. Hayashi, J. Appl. Phys. 95, 272 (2004).

[23] Note that when Eq. (3) is resolved with the estimated linewidths, a larger $\beta(\tau)$ is extracted for the dip frequency with respect to those at the peaks. This has been verified for all experimental data. The fact that smaller $\beta$ 's are observed at resonances is a strong evidence of cavityrelated dynamics, confirming further our model.

[24] The experimentally determined fitting coefficient $A$ in Eq. (1) is proportional to $\left\langle\cos (\theta)^{2}\right\rangle$, where $\theta$ is the angle between the unpolarized $\mathrm{Si}-\mathrm{NC}$ dipole emission and the polarized TM-like WGM. Therefore, $A$ takes into account the correction factor for polarization mismatch.

[25] This term, $f(\langle\omega\rangle)=\frac{\Delta \omega_{\mathrm{em}}^{2}}{\Delta \omega_{\mathrm{em}}^{2}+4\left(\langle\omega\rangle-\omega_{\mathrm{cav}}\right)^{2}}$, has been averaged for the spectral resolution of $0.25 \mathrm{~nm}$, inducing a negligible correction of $\sim 2 \%$ in the estimation of the linewidth, $\Delta \omega_{\mathrm{em}}$.

[26] M. Dovrat, Y. Oppenheim, J. Jedrzejewski, I. Balberg, and A. Sa'ar, Phys. Rev. B 69, 155311 (2004).

[27] D. Kovalev et al., Phys. Rev. Lett. 81, 2803 (1998).

[28] T. J. Kippenberg, S. M. Spillane, and K. J. Vahala, Appl. Phys. Lett. 85, 6113 (2004).

[29] Y. Akahane, T. Asano, B.-S. Song, and S. Noda, Opt. Express 13, 1202 (2005). 